\title{
A multi-wavelength pipeline for pulsar observations
}

\author{
Maura Pilia ${ }^{1}$ Alessio Trois ${ }^{1}$, Matteo Bachetti ${ }^{1}$, Alberto Pellizzoni ${ }^{1}$, \\ Elise Egron ${ }^{1}$, Maria Noemi Iacolina ${ }^{1,2}$ and Sara Loru $^{1}$ \\ ${ }^{1}$ INAF - Osservatorio Astronomico di Cagliari, \\ via della Scienza 5, I-09047 Selargius (Cagliari), Italy \\ ${ }^{2}$ ASI - Italian Space Agency - via del Politecnico snc, I-00133 Roma, Italy
}

\begin{abstract}
The Astronomical Observatory in Cagliari (OAC) is a growing facility with a group devoted to pulsar studies across the electromagnetic spectrum. Taking advantage of this expertise we have worked to provide a suite of multi-wavelength software and databases for the observations of pulsars and compact Galactic objects at the Sardinia Radio Telescope (SRT, Bolli et al. 2015, Prandoni et al. 2017).
\end{abstract}

Keywords. astronomical data bases: miscellaneous, pulsars: general

\section{Introduction}

The aim of this project is to provide the observer with a set of online tools to be accessible during the observation at SRT, in order to look in real time for high energy counterparts of the target(s) they are pointing at. As a demostrator of this package, which will be made available when SRT observations will be up to speed, two offline independent pipelines have already been tested for radio and $\gamma$-rays, and a third one is in the making for the addition of X-ray data.

\section{Outline of the Infrastructure}

A brief description follows of each pipeline, schematically illustrated in Figure 1.

Radio pipeline. Observations of pulsars and compact objects at SRT will be processed by the ROACH-2 based SARDARA backend (Melis et al., submitted). SARDARA will provide full-Stokes observations with different configurations (up to 16000 frequency channels and 16000 spectra per second) to optimally observe pulsars at all bands available at SRT (P-, L-, C- and K-band, soon also a 7-feed S-band). We have developed a python wrapper around the standard pulsar search software presto (Ransom 2001) which allows standard and accelerated search plus single pulse search for strong pulsars and transients. The pipeline is being optimised for RFI-excision given the highly polluted interference environment surrounding the telescope. Candidate selection will be partly authomatised through the application of machine learning techniques (either one that seems optimal for SRT data or a combination of more than one in order to avoid the bias of a single classification). Pulsar gating is also implemented, to improve the search for underlying diffuse emission surrounding the compact object (e.g. a pulsar wind nebula, PWN).

Gamma-ray pipeline. Online archives are available for $\gamma$-ray observations of the two $\gamma$-ray telescopes in orbit: the AGILE-GRID and Fermi-LAT. We developed a simple command line interface to the archives of the two telescopes, so that data from both can be directly downloaded on our servers for the requested position and time interval. Additional specifics such as energy-range, data quality, off-axis angle, albedo filtering, 


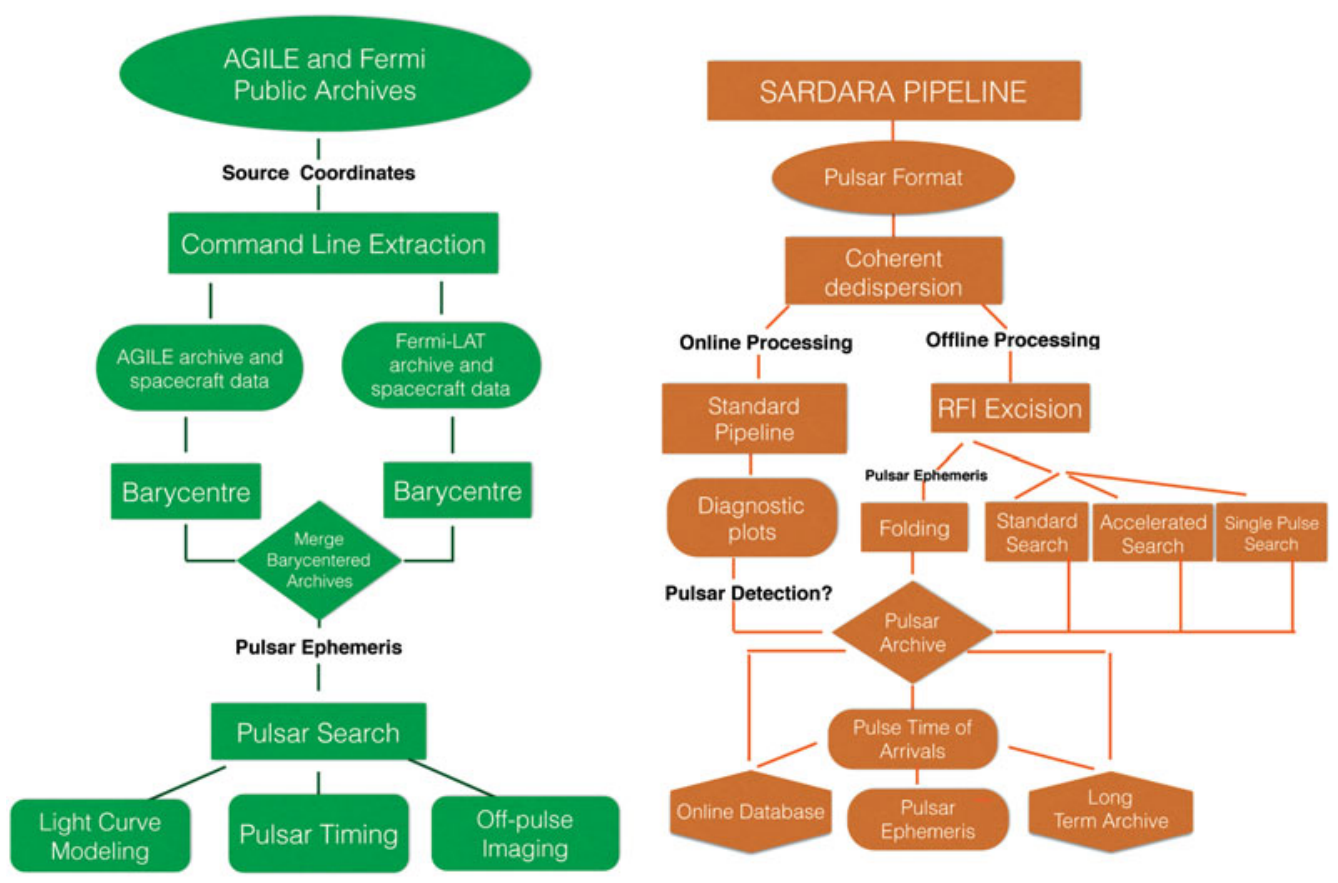

Figure 1. Outline of the radio (left) and $\gamma$-ray (right) pipelines.

can be also provided. Given the center position, the pipeline barycenters the data and, if so chosen, it can create a single fits file including the GTIs and barycentered times for the combined dataset. After barycentering is performed, standard folding can be carried out using a known ephemeris of the source (see e.g. Pellizzoni et al. 2009a), and follow-up off-pulse analysis of nebular emission (as in Pellizzoni et al. 2010). Optimised tools for exposure calculation for each satellite are being tested, as is blind search on unidentified sources.

$X$-ray pipeline. A similarly structured command-line pipeline has already been developed in-house to download and analyse NuSTAR data and is available upon request (https://gitlab.com/matteobachetti/heasarc_pipelines). Because our group is part of the Italian team responsible for the development, calibration and validation of the scientific software for the Imaging X-ray Polarimetry Explorer (IXPE, Weisskopf et al. 2016), future prospects include the development of a pipeline for the processing of IXPE public data as an important addition to our high-energy database. IXPE will be able to do polarimetry and timing analyses (time tagging accuracy of less than $100 \mu \mathrm{s}$ ), opening an important new window on pulsars and PWNe.

\section{Acknowledgements}

The project "Development of a Software Tool for the Study of Pulsars from Radio to Gamma-rays using Multi-mission Data" is supported by the Autonomous Region of Sardinia (RAS), CRP-25476.

\section{References}

Bolli, P., Orlati, A., Stringhetti, L., et al. 2015, JAI, 4, 50008.

Pellizzoni, A., Pilia, M., Possenti, A., et al. 2009, ApJ, 691, 1618.

Pellizzoni, A., Trois, A., Tavani, M., Pilia, M., et al. 2010, Science, 327, 663.

Prandoni, I., Murgia, M., \& Tarchi, A. et al. 2017, A\&A, in press.

Ransom, S. M. 2001, Ph.D. thesis, Harvard University

Weisskopf, M. C., Ramsey, B., O’Dell, S. et al. 2016 SPIE 9905, 17. 